\title{
Produção e amplitude de colheita de cultivares de nogueira-macadâmia em Itapira, São Paulo
}

\author{
Rafael Pio ${ }^{1}$, Edwaldo dos Santos Penoni², Filipe Almendagna Rodrigues ${ }^{2}$, \\ José Darlan Ramos ${ }^{1}$, Antônio Decarlos Neto ${ }^{1}$
}

\begin{abstract}
RESUMO
A nogueira-macadâmia produz frutos do tipo folículo, cuja parte comestível é a semente, com alto valor agregado no mercado internacional e com grande aceitação pelos consumidores. No Brasil, sabe-se que a época de colheita dos frutos da nogueira-macadâmia inicia-se em meados de fevereiro, porém, não se conhece o potencial produtivo dos diversos cultivares disponíveis nas condições brasileiras. O objetivo deste trabalho foi quantificar a produção e a amplitude de colheita de cultivares de nogueira-macadâmia, no município de Itapira, SP. Para o experimento, foram utilizados dez cultivares de nogueira-macadâmia (HAES 722, IAC Campinas-B, 791 Fuji, HAES 842, HAES 849, HAES 814, HAES 344, IAC 9-20X, IAC 9-20 e HAES 816), sendo quantificadas durante três safras, em Itapira, SP, o número de frutos e a massa de colheita (produção e produtividade estimada), calculando-se, posteriormente, a massa média dos frutos. Concluiu-se que a produção da nogueira-macadâmia, em Itapira, SP, inicia-se em meados de fevereiro e estendese até o final de junho. IAC 9-20 foi o cultivar mais precoce e, HAES 722, o mais tardio, enquanto o HAES 344 proporcionou a menor amplitude de colheita e 791 Fuji e HAES 849 as maiores. Os cultivares IAC 9-20X, IAC 9-20 e HAES 816 apresentaram o maior desempenho produtivo.
\end{abstract}

Palavras-chave: Macadamia integrifolia, amêndoa, seleção de cultivares, produção.

\section{ABSTRACT}

\section{Production and breadth of harvest of walnut macadamia in Itapira, São Paulo, Brazil}

Macadamia nuts achieve high values on the international market with widespread acceptance by consumers. In Brazil, harvesting of macadamia fruits starts in mid february, but it is not known the productive potential of different cultivars available in Brazilian conditions. The objective of this study was to quantify the production and extent of harvest of macadamia cultivars in Itapira, São Paulo State. Ten macadamia cultivars (HAES 722, IAC Campinas-B, 791 Fuji, HAES 842, HAES 849, HAES 814, HAES 344, IAC 9-20X, IAC 9-20 and HAES 816) were used in the experiment. The following characteristics were evaluated during three harvest seasons in Itapira-SP: the number of fruits and harvest mass (production and estimated yield), then calculating the average mass of fruits. It was concluded that the production of macadamia nuts in Itapira-SP began in mid February and runs until the end of June. Cultivar IAC 9-20 was the earliest and HAES 722 the latest, whereas HAES 344 provided the lowest range of harvest and 791 Fuji and HAES 849 the greatest. IAC 9-20X, IAC 9-20 and HAES 816 showed the highest growth performance.

Key words: Macadamia integrifolia, nut, selection of cultivars, production

\footnotetext{
Recebido para publicação em 05/10/2011 e aprovado em 22/11/2012.

Engenheiro-Agrônomo, Doutor. Departamento de Agricultura, Universidade Federal de Lavras, Caixa Postal 3037, 37200-000, Lavras, Minas Gerais, Brasil. rafaelpio@dag.ufla.br (autor para correspondência); darlan@dag.ufla.br; adecarlos@pop.com.br

${ }^{2}$ Engenheiro-Agrônomo, Mestre. Programa de Pós-graduação em Fitotecnia, Universidade Federal de Lavras, Caixa Postal 3037, 37200-000, Lavras, Minas Gerais, Brasil. espenoni@bol.com.br; filipealmendagna@yahoo.com.br
} 


\section{INTRODUÇÃO}

A nogueira-macadâmia (Macadamia integrifolia Maiden \& Betche), originária das florestas subtropicais australianas, é, atualmente, cultivada em diversos países, a exemplo do Brasil. Essa fruteira apresenta boas perspectivas de comercialização, pois produz nozes de alto valor agregado no mercado internacional, pelas características intrínsecas de suas nozes (Bastos et al., 2006; Dalastra et al., 2010).

O óleo da noz macadâmia é rico em ácidos graxos monoinsaturados (Borompichaichartkul et al., 2009), com propriedades químicas similares às do azeite de oliva (Oliveira et al., 2012; Silva et al., 2012). Freitas \& Naves (2010) analisaram a percentagem de ácidos graxos de nozes verdadeiras e sementes comestíveis de diferentes espécies, e verificaram a presença dos ácidos palmítico $(8,88 \%)$, esteárico $(4,26 \%)$, oleico $(58,51 \%)$, linoleico $(1,81 \%)$ e linolênico (2,58\%), em nozes de nogueira-macadâmia.

Apesar de esta fruteira ser nativa da Austrália, foi somente após sua introdução nos EUA que os trabalhos de seleção varietal foram iniciados. No Havaí, foram lançados os cultivares de nogueira-macadâmia da série HAES, pelo Hawaii Agricultural Experiment Station, como 'Pahala' (HAES 788), 'Kau' (HAES 344), 'Keauhou' (HAES 246), 'Mauka' (HAES 741), 'Ikaika' (HAES 333), 'Kakea' (HAES 508), 'Keaau' (HAES 660), 'Makai' (HAES 800), 'Keauhou' (HAES 224), dentre outras de menor importância comercial (Peace et al., 2005).

No Brasil, os trabalhos de melhoramento genético com a nogueira-macadâmia iniciaram-se pelo Instituto Agronômico (IAC), e resultaram no lançamento de algumas seleções para plantios comerciais (Sobierajski et al., 2006). Apesar do número de cultivares disponíveis no Estado de São Paulo, maior produtor nacional, apenas 'Keauhou', 'Mauka', 'IAC Campinas-B' e 'IAC 9-20' são cultivados em maior escala (Barbosa et al., 2003). Acredita-se que a baixa diversificação de cultivares possa estar atrelada ao fato de a exploração da nogueira-macadâmia ser recente, no Brasil, e as informações sobre as tecnologias de cultivo estarem baseadas nos resultados de pesquisa da Austrália e Havaí (Machado Neto \& Moryia, 2010).

A produção da nogueira-macadâmia é quantificada em nozes em casca (casca e amêndoa) (Sobierajski et al., 2006). Em trabalho realizado no Kenya, Gitonga et al. (2009) apontaram que uma planta adulta com 15 anos de idade pode chegar a produzir $40 \mathrm{~kg}$ de nozes em casca. No Brasil, a época de colheita dos frutos da nogueiramacadâmia inicia-se no mês de fevereiro (Sobierajski et al., 2007); sabe-se que há diferença na quantidade de ácidos graxos entre os cultivares (Maro et al., 2012), mas se desconhece o potencial produtivo da nogueirasmacadâmias, necessitando-se quantificar a produção dos cultivares disponíveis nas condições brasileiras, para, assim, obterem-se pomares altamente tecnificados, com elevada produção.

Este trabalho objetivou quantificar a produção e a amplitude de colheita de dez cultivares de nogueiramacadâmia, em Itapira, SP.

\section{MATERIAL E MÉTODOS}

Foi selecionado um terreno plano e homogêneo, na área de produção da empresa Macadâmia Brasilis LTDA, localizada no município de Itapira, SP, na região leste paulista, para a realização do experimento. O clima da região, segundo classificação de Köppen, é mesotérmico de inverno seco (Cwa), comumente chamado de tropical de altitude, com temperatura anual média de $18^{\circ} \mathrm{C}$ e precipitação média anual de $1.300 \mathrm{~mm}$ (Pio et al., 2007; Bettiol Neto et al., 2011, Chagas et al., 2012). A propriedade situa-se em uma altitude de 665 a $675 \mathrm{~m}$. O solo no local do experimento pertence ao grande grupo Litosol, fase substrato filito-xisto (Embrapa, 2006).

$\mathrm{O}$ experimento foi avaliado em esquema de parcela subdividida no tempo, sendo os cultivares as parcelas e os três anos de avaliação (safras) as subparcelas, com quatro blocos e cinco plantas por parcela, sendo avaliadas apenas as três plantas centrais.

No talhão selecionado, havia dez cultivares de nogueira-macadâmia em espaçamento $8 \times 6 \mathrm{~m}$, sendo as linhas compostas por cinco plantas de cada cultivar. Os cultivares HAES 722, IAC Campinas-B, 791 Fuji, HAES 842, HAES 849, HAES 814, HAES 344 ('Kau'), IAC 9-20X, IAC 9-20 e HAES 816 foram enxertados no porta-enxerto 'Aloha' e levados a campo em 2004. As plantas não foram irrigadas, mas foram adubadas nos três anos de avaliação, com o formulado NPK 18-06-24, nos meses de janeiro e novembro, com as doses de 200 e $500 \mathrm{~g}$, respectivamente, por planta.

A primeira safra, ocorrida em 2008, foi desconsiderada, na avaliação, pela pouca produção de frutos. Nas três safras seguintes $(2009,2010$ e 2011), foram marcados o início e o término da colheita. Durante o período de safra, realizaram-se coletas semanais dos frutos inteiros caídos no solo (carpelo, casca e amêndoa) e que se localizavam na projeção da copa da planta. Os frutos foram cuidadosamente separados em sacos identificados e levados para o barracão da empresa, onde foram removidos os carpelos, restando apenas a noz em casca (casca e amêndoa). A cada colheita, quantificou-se o número de frutos e a massa da colheita, calculando-se, posteriormente, a massa média dos frutos. No término da safra, calculou-se a produção média das plantas, quantificando a massa média dos frutos, o número médio de frutos e a produtividade média estimada. 
A normalidade dos dados foi analisada pelo teste de Shapiro-Wilks e, a homocedasticidade, pelo teste de Hartley. Os dados de produção foram submetidos à análise de variância e as médias agrupadas pelo teste de ScottKnott, a 5\% de probabilidade, pelo programa estatístico SISVAR 4.3. (Ferreira, 2011).

\section{RESULTADOS E DISCUSSÃO}

A colheita dos frutos da maioria dos cultivares de nogueira-macadâmia iniciou-se na primeira semana do mês de março do ano de 2009, à exceção da colheita do cultivar HAES 816, que se iniciou na segunda quinzena de abril. Os cultivares HAES 344 e IAC 9-20 produziram somente até o início de maio, enquanto outros cultivares, como HAES 722, IAC Campinas-B, 791 Fuji, HAES 849, HAES 814 e HAES 816, produziram até o final do mês de junho. No ano de 2010, as colheitas anteciparam-se, sendo os cultivares mais precoces o IAC Campinas-B, o HAES 814 e o IAC 9-20, pois iniciaram o período de safra no início do mês de fevereiro. Porém, HAES 344 e IAC 9-20 finalizaram a produção no início de maio, enquanto outros cultivares, como HAES 722, 791 Fuji, HAES 849, HAES 814 e HAES 816 produziram até o final de junho (Tabela 1).

Em 2011, o período de safra do cultivar IAC 9-20 começou no início do mês de fevereiro; os cultivares HAES 722 e HAES 816 iniciaram o período de safra somente no início de março, enquanto os demais cultivares no início da segunda quinzena de fevereiro. Novamente HAES 344 e IAC 9-20 finalizaram a produção no início de maio e HAES 722, 791 Fuji, HAES 849, HAES 814 e HAES 816 produziram até o final de junho (Tabela 1).

Com esses resultados foi possível verificar que os cultivares de nogueira-macadâmia produzem somente no primeiro semestre do ano, nas condições de Itapira, SP, e

Tabela 1.Época e amplitude de colheita de dez cultivares de nogueira-macadâmia em três safras, em Itapira-SP. Lavras, UFLA, 2011

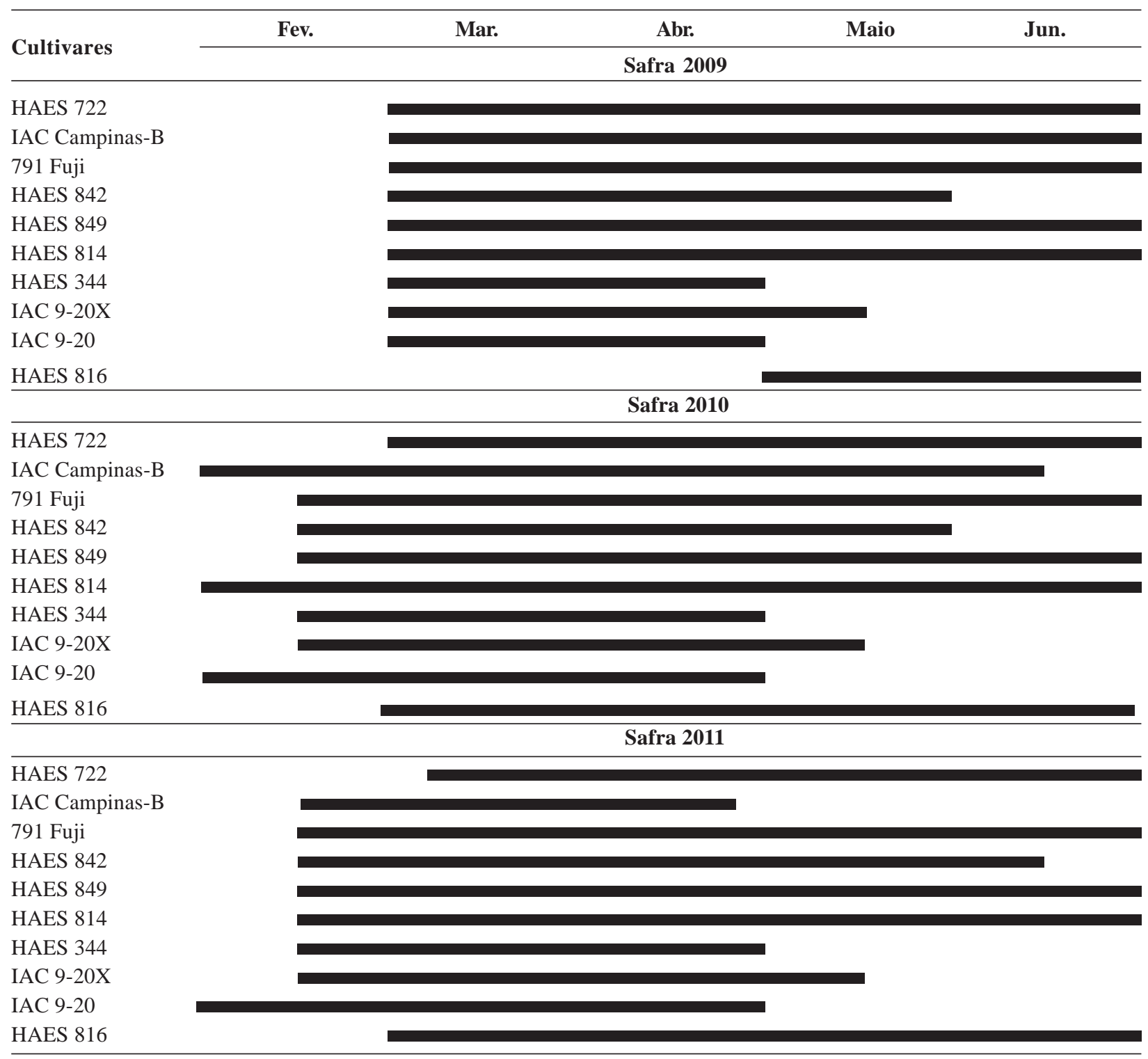


Tabela 2. Número médio de frutos, produção média, produtividade média estimada e massa média das nozes em casca de dez cultivares de nogueira-macadâmia em três safras, em Itapira-SP. Lavras, UFLA, 2011

\begin{tabular}{|c|c|c|c|}
\hline \multirow{2}{*}{ Cultivares } & Safra 2009* & Safra 2010 & Safra 2011 \\
\hline & \multicolumn{3}{|c|}{$\mathrm{N}^{0}$ médio de frutos } \\
\hline HAES 722 & $436,99 \mathrm{Bc}$ & $557,64 \mathrm{Bd}$ & $722,84 \mathrm{Ad}$ \\
\hline IAC Campinas-B & $876,90 \mathrm{Bb}$ & $982,45 \mathrm{Bb}$ & $1.762,37 \mathrm{Ab}$ \\
\hline 791 Fuji & $533,29 \mathrm{Cc}$ & $823,65 \mathrm{Bc}$ & $1.612,14 \mathrm{Ab}$ \\
\hline HAES 842 & $493,45 \mathrm{Cc}$ & $877,45 \mathrm{Bc}$ & $1.603,26 \mathrm{Ab}$ \\
\hline HAES 849 & $350,36 \mathrm{Cd}$ & $577,74 \mathrm{Bd}$ & $1.356,13 \mathrm{Ac}$ \\
\hline HAES 814 & $501,93 \mathrm{Bc}$ & $610,53 \mathrm{Bd}$ & $1.186,87 \mathrm{Ac}$ \\
\hline HAES 344 & $473,62 \mathrm{Bc}$ & $858,71 \mathrm{Bd}$ & $1.302,23 \mathrm{Ac}$ \\
\hline IAC 9-20X & $1.312,65 \mathrm{Ba}$ & $1.385,24 \mathrm{Ba}$ & $1.913,93 \mathrm{Aa}$ \\
\hline IAC 9-20 & $652,12 \mathrm{Cc}$ & $1.263,18 \mathrm{Ba}$ & $1.974,23 \mathrm{Aa}$ \\
\hline HAES 816 & $245,45 \mathrm{Cd}$ & $972,33 \mathrm{Bb}$ & $1.969,22 \mathrm{Aa}$ \\
\hline CV (\%) Parcela (cultivar) & & 24,48 & \\
\hline \multirow[t]{2}{*}{ CV (\%) Subparcela (ano) } & & 9,45 & \\
\hline & \multicolumn{3}{|c|}{ Produção média (kg) } \\
\hline HAES 722 & $3,45 \mathrm{Bc}$ & $4,37 \mathrm{Bd}$ & $5,54 \mathrm{Ae}$ \\
\hline IAC Campinas-B & $5,76 \mathrm{Bb}$ & $6,27 \mathrm{Bc}$ & $10,70 \mathrm{Ac}$ \\
\hline 791 Fuji & $5,18 \mathrm{Cb}$ & $7,74 \mathrm{Bb}$ & $13,55 \mathrm{Ab}$ \\
\hline HAES 842 & $3,68 \mathrm{Cc}$ & $6,40 \mathrm{Bc}$ & $11,04 \mathrm{Ac}$ \\
\hline HAES 849 & $2,30 \mathrm{Cd}$ & $3,66 \mathrm{Bd}$ & 8,16 Ad \\
\hline HAES 814 & $3,14 \mathrm{Bc}$ & $3,66 \mathrm{Bd}$ & $6,74 \mathrm{Ae}$ \\
\hline HAES 344 & $3,71 \mathrm{Bc}$ & $4,50 \mathrm{Bd}$ & $9,46 \mathrm{Ac}$ \\
\hline IAC $9-20 X$ & $9,65 \mathrm{Ba}$ & $10,15 \mathrm{Ba}$ & $15,14 \mathrm{Aa}$ \\
\hline IAC 9-20 & $4,86 \mathrm{Cb}$ & $9,25 \mathrm{Ba}$ & $15,08 \mathrm{Aa}$ \\
\hline HAES 816 & $1,95 \mathrm{Cd}$ & $7,60 \mathrm{Bb}$ & 15,25 Аа \\
\hline CV (\%) Parcela (cultivar) & & 19,97 & \\
\hline \multirow[t]{2}{*}{ CV (\%) Subparcela (ano) } & & 9,87 & \\
\hline & \multicolumn{3}{|c|}{ Produtividade média estimada $\left(\mathrm{kg} \mathrm{ha}^{-1}\right)$} \\
\hline HAES 722 & $718,74 \mathrm{Bc}$ & $911,44 \mathrm{Bd}$ & $1.155,19 \mathrm{Ae}$ \\
\hline IAC Campinas-B & $1.201,02 \mathrm{Bb}$ & $1.306,23 \mathrm{Bc}$ & $2.229,13 \mathrm{Ac}$ \\
\hline 791 Fuji & $1.080,19 \mathrm{Cb}$ & $1.613,51 \mathrm{Bb}$ & $2.822,87 \mathrm{Ab}$ \\
\hline HAES 842 & $766,65 \mathrm{Cc}$ & $1.333,31 \mathrm{Bc}$ & 2.301,00 Ac \\
\hline HAES 849 & $479,16 \mathrm{Cd}$ & $762,48 \mathrm{Bd}$ & $1.699,97 \mathrm{Ad}$ \\
\hline HAES 814 & $654,15 \mathrm{Bc}$ & $763,53 \mathrm{Bd}$ & $1.405,19 \mathrm{Ae}$ \\
\hline HAES 344 & $722,90 \mathrm{Bc}$ & $937,48 \mathrm{Bd}$ & $1.970,80 \mathrm{Ac}$ \\
\hline IAC $9-20 X$ & $2.010,38 \mathrm{Ba}$ & $2.114,55 \mathrm{Ba}$ & $3.154,11 \mathrm{Aa}$ \\
\hline IAC $9-20$ & $1.012,48 \mathrm{Cb}$ & $1.927,05 \mathrm{Ba}$ & $3.141,61 \mathrm{Aa}$ \\
\hline HAES 816 & $406,24 \mathrm{Cd}$ & $1.583,31 \mathrm{Bb}$ & $3.177,72 \mathrm{Aa}$ \\
\hline$\overline{\mathrm{CV}(\%) \text { Parcela (cultivar) }}$ & & 19,97 & \\
\hline CV (\%) Subparcela (ano) & & 9,87 & \\
\hline
\end{tabular}

\begin{tabular}{lc}
\hline & Massa média das nozes em casca, nas três safras $(\mathbf{g})$ \\
\hline HAES 722 & $7,82 \mathrm{~b}$ \\
IAC Campinas-B & $6,32 \mathrm{c}$ \\
791 Fuji & $9,68 \mathrm{a}$ \\
HAES 842 & $7,20 \mathrm{~b}$ \\
HAES 849 & $6,34 \mathrm{c}$ \\
HAES 814 & $6,06 \mathrm{c}$ \\
HAES 344 & $7,60 \mathrm{~b}$ \\
IAC 9-20X & $7,55 \mathrm{~b}$ \\
IAC 9-20 & $7,48 \mathrm{~b}$ \\
HAES 816 & $7,84 \mathrm{~b}$ \\
\hline CV $(\%)$ & 14,83 \\
\hline
\end{tabular}

* Médias seguidas pela mesma letra maiúscula, na linha, e minúscula, na coluna, não diferem significativamente entre si, pelo teste ScottKnott, a $5 \%$ de probabilidade. 
diferem entre si quanto ao início e término do período de colheita. Os resultados estão de conformidade com os obtidos por Sobierajski et al. (2007), que verificaram que a colheita de nogueira-macadâmia inicia-se no mês de fevereiro, nas condições de Jundiaí, SP.

No Brasil, normalmente, o amadurecimento dos frutos da nogueira-macadâmia ocorre no outono, principalmente entre os meses de março a maio, e a colheita dá-se após a queda ao solo, por coleta manual (Sobierajski et al., 2007).

Em relação aos anos de avaliação, a colheita da primeira safra (2009) foi iniciada somente no mês de março e a amplitude de colheita foi mais curta, em relação à colheita dos demais anos. Este fato pode ser devido às plantas ainda serem jovens.

Quanto ao desempenho produtivo, a análise estatística revelou que houve interação entre os cultivares e as três safras de avaliação, para o número médio de frutos, produção média e produtividade média estimada. No caso da massa média das nozes, ocorreu diferença estatística somente entre os cultivares (Tabela 2).

Os cultivares 791 Fuji, HAES 842, HAES 849, IAC 9-20 e HAES 816 apresentaram diferença estatística para o número de frutos, produção e produtividade estimada, entre as três safras avaliadas, enquanto os demais cultivares não registraram diferenças estatística, entre os desempenhos produtivos, em relação às duas primeiras safras (Tabela 2).

Na safra de 2009, o cultivar IAC 9-20X registrou maior número de frutos e, consequentemente, maior produção por planta e produtividade média estimada, de nozes em casca $\left(9,65 \mathrm{~kg}\right.$ e $2.010,38 \mathrm{~kg} \mathrm{ha}^{-1}$ ) (Tabela 2). Na segunda safra, novamente esse cultivar e o 'IAC 9-20' destacaramse entre os demais. Na terceira safra, esses dois cultivares, juntamente com 'HAES 816', apresentaram maior número de frutos, maior produção e produtividade estimada (Tabela 2).

Normalmente, a quantidade média de nozes em casca produzidas em pomares de nogueira-macadâmia é de 1,5 $\mathrm{kg}$ por árvore, em plantas com cinco anos de idade, $4 \mathrm{~kg}$ com seis anos, $6,5 \mathrm{~kg}$ com sete anos, $8 \mathrm{~kg}$ com oito anos, $12 \mathrm{~kg}$ com nove anos e $14 \mathrm{~kg}$ com dez anos (Gabelini, 2009). Pelos resultados alcançados neste trabalho, verificou-se que, na primeira safra de avaliação, ocorrida no ano de 2009, todos os cultivares superaram a média produtiva de $1,5 \mathrm{~kg} \mathrm{planta}^{-1}$, relatada pela literatura. Fato marcante, que se deve aos três cultivares de maior desempenho produtivo ('IAC 9-20X', 'IAC 9-20' e 'HAES 816'), que atingiram média produtiva acima de $15 \mathrm{~kg}$ planta $^{-1}$, já aos sete anos de idade, produção esta alcançada somente em pomares com dez anos de idade (Gabelini, 2009).

De acordo com Gabelini (2009), a seleção dos cultivares de nogueira-macadâmia é de extrema importância para a formação de um pomar produtivo, e será determinada de acordo com clima, tipo de solo, densidade, topografia e forma de manejo.

Houve diferença significativa para massa média de nozes em casca, entre os cultivares. O cultivar 791 Fuji apresentou-se com destaque, em relação aos outros, estudados (Tabela 2). Os valores encontrados para a massa média das nozes em casca diferiram, ligeiramente, dos resultados encontrados por Penoni et al. (2011), que, no caso, selecionaram 100 nozes por cultivar, para a quantificação das massas de nozes em casca. Neste trabalho, todas as nozes foram contadas e pesadas e, no final, obteve-se o valor médio da massa. Mesmo assim, percebe-se pouca variação dos valores numéricos, a exemplo dos valores do cultivar 791 Fuji, para o qual se obteve valor médio da massa de $9,68 \mathrm{~g}$, ao passo que Penoni et al. (2011) registraram 9,59 $\mathrm{g}$.

Como a produção da nogueira-macadâmia é quantificada em nozes em casca (casca e amêndoa) (Sobierajski et al., 2006), quanto maior a massa da noz em casca, maior será o retorno econômico obtido pelo produtor. Contudo, mais estudos devem ser realizados, visando a identificar cultivares que, além de serem mais produtivos, apresentem frutos de maior calibre e maior massa de amêndoas e de nozes em casca.

\section{CONCLUSÃO}

Concluiu-se que a produção da nogueira-macadâmia, em Itapira, SP, inicia-se em meados de fevereiro e estendese até o final de junho. O cultivar IAC 9-20 é o mais precoce e HAES 722 o mais tardio, enquanto HAES 344 proporciona a menor amplitude de colheita e, 791 Fuji e HAES 849, as maiores. Os cultivares IAC 9-20X, IAC 9-20 e HAES 816 apresentaram o maior desempenho produtivo.

\section{REFERÊNCIAS}

Barbosa W, Pommer CV, Ribeiro MD, Veiga RFA \& Costa AA (2003) Distribuição geográfica e diversidade varietal de frutíferas e nozes de clima temperado no Estado de São Paulo. Revista Brasileira de Fruticultura, 25:341-344.

Bastos DC, Pio R, Araújo JPC \& Scarpare Filho JA (2006) Efeito do ácido indolbutírico na propagação por estacas de dois cultivares de macadâmia (Macadamia integrifolia Maiden \& Betche). Científica Rural, 11:120-125.

Bettiol Neto JE, Pio R, Sanches J, Chagas EA, Cia P, Chagas PC \& Antoniali S (2011) Produção e atributos de qualidade de cultivares de marmeleiro na região Leste paulista. Revista Brasileira de Fruticultura, 33:1035-1042.

Borompichaichartkul C, Luengsode K, Chinprahast N \& Devahastin S (2009) Improving quality of macadamia nut (Macadamia integrifolia) through the use of hybrid drying process. Journal of Food Engineering, 93:348-353.

Chagas EA, Chagas PC, Pio R, Bettiol Neto JE, Sanches J, Carmo AS, Cia P, Pasqual M \& Carvalho AS (2012) Produção e atributos de qualidade de cultivares de macieira nas condições subtropicais da região Leste paulista. Ciência Rural, 42:1764-1769. 
Dalastra IM, Pio R, Entelmann FA, Werle T, Uliana MB \& Scarpare Filho JA (2010) Germinação de sementes de nogueiramacadâmia submetidas à incisão e imersão em ácido giberélico. Ciência e Agrotecnologia, 34:641-645.

Empresa Brasileira de Pesquisa Agropecuária - Embrapa (2006) Centro Nacional de Pesquisa de Solos. Sistema Brasileiro de Classificação de Solos. $2^{\circ}$ ed. Rio de Janeiro, Embrapa Solos. 306p.

Ferreira DF (2011) Sisvar: A computer statistical analysis system. Ciência e Agrotecnologia, 35:1039-1042.

Freitas JB \& Naves MMV (2010) Composição química de nozes e sementes comestíveis e sua relação com a nutrição e saúde. Revista Nutrição, 23:269-279.

Gabelini RCBS (2009) Reguladores vegetais na emergência e desenvolvimento de plantas de macadamia (Macadamia integrifolia Maiden \& Betche). Tese de Doutorado. Universidade Estadual Paulista, Botucatu. 94p.

Gitonga LN, Muigai AWT, Kahangi EM, Ngamau K \& Gichuki ST (2009) Status of macadamia production in Kenya and the potential of biotechnology in enhancing its genetic improvement. Journal of Plant Breeding and Crop Science, 1:49-59.

Machado Neto NB \& Moryia AT (2010) Variability in Macadamia integrifolia by RAPD markers. Crop Breeding and Applied Biotechnology, 10:266-270.

Maro LAC, Pio R, Penoni ES, Oliveira MC, Prates FC, Lima LCO \& Cardoso MG (2012) Caracterização química e perfil de ácidos graxos em cultivares de nogueira-macadâmia. Ciência Rural, 42:2166-2171
Oliveira MC, Ramos JD, Pio R \& Cardoso MG (2012) Características fenológicas e físicas e perfil de ácidos graxos em oliveiras no sul de Minas Gerais. Pesquisa Agropecuária Brasileira, 47:30-35.

Peace CP, Allan P, Vithanage V, Turnbull C \& Carroll BJ (2005) Genetic relationships amongst macadamia varieties grown in South Africa as assessed by RAF markers. South African Journal of Plant \& Soil, 22:71-75.

Penoni ES, Pio R, Rodrigues FA, Maro LAC \& Costa FC (2011) Análise de frutos e nozes de cultivares de nogueira-macadâmia. Ciência Rural, 41:2080-2083.

Pio R, Dall'Orto FAC, Barbosa W, Chagas EA, Ojima \& Cia P (2007) Produção de cultivares de nespereira na região Leste paulista. Pesquisa Agropecuária Brasileira, 42:1053-1056.

Silva LFO, Oliveira AF, Pio R, Alves TC \& Zambon CR (2012) Variação na qualidade do azeite em cultivares de oliveira. Bragantia, 71:202-209.

Sobierajski GR, Barbosa W, Bettiol Neto JE, Chagas EA \& Dall'Orto FAC (2007) Caracterização dos estágios fenológicos em sete cultivares e seleções de nogueira-macadâmia. Revista Brasileira de Fruticultura, 29:690-694.

Sobierajski GR, Francisco VLFS, Rocha P, Ghilardi AA \& Maia ML (2006) Noz-macadâmia: produção, mercado e situação no Estado de São Paulo. Informações Econômicas, 36:25-36. 